HEFAT2007

$5^{\text {th }}$ International Conference on Heat Transfer, Fluid Mechanics and Thermodynamics

Sun City, South Africa

Paper number: K2

\title{
RENDERING THE TRANSIENT HOT WIRE EXPERIMENTAL METHOD TO DUAL-PHASE APPLICATIONS
}

\author{
Vadasz, P. \\ Department of Mechanical Engineering, \\ Northern Arizona University, \\ Flagstaff, AZ 86004, \\ U. S. A., \\ E-mail: peter.vadasz@nau.edu
}

\begin{abstract}
The Transient Hot Wire method is well established as the most accurate, reliable and robust technique for evaluating the thermal conductivity of fluids and solids. Unfortunately its direct application to dual-phase systems such as solid suspensions in fluids or porous media cannot be supported by the very principles and methodology underlying this method. The derivation of possible ways of rendering the transient hot wire method to dual-phase applications including the development of validity criteria for such applications is proposed and discussed.
\end{abstract}

\section{INTRODUCTION}

The application of the Transient Hot Wire (THW) experimental method for the measurement of thermal conductivity of materials is presently limited to homogeneous and single phase materials because its simple and elegant theoretical expression on which it is based cannot be extended without associated corrections to dual-phase and heterogeneous systems. This is a first preliminary attempt at addressing the latter limitations by developing simple expressions for porting the THW method of experimental determination of thermal conductivity to dual-phase applications. Corrections to the THW method in the form presented by Vadasz (2006) are not sufficiently simple because they are expressed in terms of a ratio of infinite power series rather than a simple expression as in the single-phase case. The Dual-Phase systems that benefit from these results are nanofluid suspensions, metal foams (porous media), insulating foams (porous media), two immiscible liquids, composite solids (not a two-phase system but the method will nevertheless be applicable to such systems too).

A contextual notation is being introduced here to distinguish between dimensional and dimensionless variables and parameters. It implies that an asterisk subscript identifies dimensional values only when ambiguity arises when the asterisk is omitted. For example $x_{*}$ is the dimensional horizontal coordinate, while $x$ is its corresponding dimensionless counterpart. However $k_{s}$ is the effective solid phase thermal conductivity, a dimensional parameter that appears without an asterisk subscript without causing ambiguity.

\section{CONCEPTS AND METHODS}

Inherent assumptions for the existence of an effective thermal conductivity for dual-phase systems such as nanofluidsuspensions (Eastman et al. 2001, Lee et al. 1999, Choi et al. 2001, Xuan \& Li 2000) or porous insulating foams (Coquard \& Baillis 2006, Coquard et al. 2006) having properties that are similar to homogeneous materials are being made even without mentioning them. The practice of using the terminology "effective thermal conductivity" over the years yielded a "tradition" of not even challenging its existence - a natural internalization but rather incorrect. A direct result of this practice is the obvious application of single-phase measurements methods to dual-phase systems.

\section{Measuring temperature in a dual-phase system via the Transient Hot Wire method \\ Homogeneous Fluids and Solids}

The THW method is well established as the most accurate, reliable and robust technique (Hammerschmidt \& Sabuga, 2000) for evaluating the thermal conductivity of fluids (De Groot, Kestin \& Sookiazian 1974, Healy, De Groot \& Kestin 1976, Kestin \& Wakeham 1978) and solids (Assael et al., 2002). It replaced the steady state methods primarily because of the difficulty to determine that steady state conditions haven indeed been established and for fluids the difficulty in preventing the occurrence of natural convection and consequently the difficulty in eliminating the effects of natural convection on the heat flux. The THW method consists in principle of determining the thermal conductivity of a selected material/fluid by observing the rate at which the temperature of 
a very thin platinum (or alternatively tantalum) wire $(5 \mu \mathrm{m}$ $80 \mu \mathrm{m}$ in diameter) increases with time after a step change in voltage has been applied to it. The platinum (tantalum) wire is embedded vertically in the selected material/fluid (see Figure 1) and serves as a heat source as well as a thermometer. The temperature of the wire is established by measuring its electrical resistance, the latter being related to the temperature via a relationship of the form (Bentley, 1984)

$R_{w}=R_{o}\left[1+\beta\left(T-T_{o}\right)\right]$

A quadratic term is usually included too in eq. (1) but the linear part is already very accurate and the quadratic term ads only $0.4 \%$ to the resistance value over a wide temperature change of

$100^{\circ} \mathrm{C}$ and $0.004 \%$ over a temperature change of $10^{\circ} \mathrm{C}$ (Bentley, 1984). The requirement for a very thin $(5 \mu \mathrm{m}$ $80 \mu \mathrm{m}$ in diameter) platinum/tantalum wire is due to the need to obtain a uniform temperature across the cross section of the wire in a time scale that is substantially shorter than the time scale of thermal diffusion to the neighboring fluid. For platinum having a thermal diffusivity of $\alpha_{P_{t}}=2.6 \times 10^{-5} \mathrm{~m}^{2} / \mathrm{s}$ (Martinsos et al. 2001) and a micrometer size wire radius $\left(4.81 \times 10^{-5} \mathrm{~m}\right)$ the transient within the wire will disappear within $\sim 0.1 \mathrm{~ms}$, and therefore the readings that are being taken at times that are much longer than $0.1 \mathrm{~ms}(t>>0.1 \mathrm{~ms})$ correspond to a uniform temperature over the wire's cross section. A Wheatstone bridge is used to measure the electrical resistance $R_{w}$ of the wire (see Figure 1). The electrical resistance of a potentiometer $R_{3}$ is adjusted until the reading of the galvanometer $G$ shows zero current. When the bridge is balanced as indicated by a zero current reading on the galvanometer $\mathrm{G}$, the value of $R_{w}$ can be established from the known electrical resistances $R_{1}, R_{2}$ and $R_{3}$ by using the balanced Wheatstone bridge relationship $R_{w}=R_{1} R_{2} / R_{3}$. While the application of the method to solids and gases is straightforward its corresponding application to electrically conducting liquids needs further attention. The experiments conducted in nano-fluid suspensions listed above (Eastman et al. 2001, Lee et al. 1999, Choi et al. 2001, Xuan \& Li 2000) used a thin electrical insulation coating layer to cover the platinum wire instead of using the bare metallic wire, a technique developed by Nagasaka \& Nagashima (1981). The latter is aimed at preventing problems such as electrical current flow through the liquid causing ambiguity of the heat generation in the wire. Alternatively, Assael et al. (2004) used tantalum wires, which were anodized in situ to form a coating layer of tantalum pentoxide $\left(\mathrm{Ta}_{2} \mathrm{O}_{5}\right)$, which is an electrical insulator. Because of the very small diameter (micrometer size) and high thermal conductivity of the platinum wire the latter can be regarded as a line source in an otherwise infinite cylindrical medium (Figure 2). The rate of heat generated per unit length $\left(l_{*}\right)$ of platinum wire is therefore $\dot{q}_{l^{*}}=i V / l_{*} \mathrm{~W} \cdot \mathrm{m}^{-1}$, where $i$ is the electric current flowing through the wire and $V$ is the voltage drop across the wire.

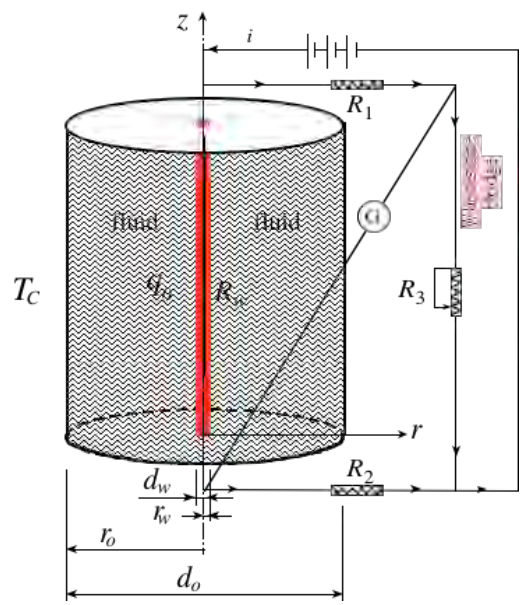

Figure 1 Typical schematic setup for a Transient Hot Wire experiment in a pure fluid.

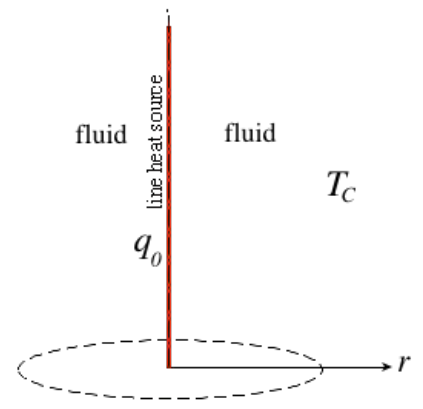

Figure 2 The line heat source analytical problem underlying the Transient Hot Wire method.

Solving for the radial heat conduction due to this line heat source leads to a temperature solution in the following closed form that can be expanded in an infinite series as follows

$T=\frac{\dot{q}_{l^{*}}}{4 \pi k} E i\left(\frac{r_{*}^{2}}{4 \alpha t_{*}}\right)=$

$\frac{\dot{q}_{l^{*}}}{4 \pi k}\left[-\gamma_{E u}+\ln \left(\frac{4 \alpha t_{*}}{r_{*}^{2}}\right)+\frac{r_{*}^{2}}{4 \alpha t_{*}}-\frac{r_{*}^{4}}{64 \alpha^{2} t_{*}^{2}}+\frac{r_{*}^{6}}{1152 \alpha^{3} t_{*}^{3}}-\ldots.\right]$

where $\operatorname{Ei}(\bullet)$ represents the exponential integral function, and $\gamma_{E u}=\ln \left(\sigma_{E u}\right)=0.5772156649$ is Euler's constant. For a line heat source embedded in a cylindrical cell of infinite radial extent and filled with the test fluid one can use the approximation $r_{*}^{2} / 4 \alpha t_{*}<<1$ in equation (2) to truncate the infinite series and yield

$T \approx \frac{\dot{q}_{l^{*}}}{4 \pi k}\left[-\gamma_{E u}+\ln \left(\frac{4 \alpha t_{*}}{r_{*}^{2}}\right)+O\left(\frac{r_{*}^{2}}{4 \alpha t_{*}}\right)\right]$

Equation (3) reveals a linear relationship, on a logarithmic time scale, between the temperature and time. Therefore, one way of evaluating the thermal conductivity is from the slope of this relationship evaluated at $r_{*}=r_{w^{*}}$, for example, $r_{w^{*}}$ being the radius of the platinum wire. However the latter needs the knowledge of the thermal diffusivity, $\alpha$, of the fluid. 
Alternatively one may evaluate $k$ by using any two readings of temperature $T_{1}$ and $T_{2}$ recorded at times $t_{1^{*}}$ and $t_{2^{*}}$ respectively. The temperature difference $\left(T_{2}-T_{1}\right)$ can then be approximated by using equation (3), in the form

$$
\left(T_{2}-T_{1}\right) \approx \frac{i V}{4 \pi k l_{*}}\left[\ln \left(\frac{t_{2^{*}}}{t_{1^{*}}}\right)\right]
$$

where we replaced the heat source with its explicit dependence on $i, V$ and $l_{*}$, i.e. $\dot{q}_{l^{*}}=i V / l_{*}$. From equation (4) one can express the thermal conductivity $k$ explicitly in the form

$$
k \approx \frac{i V}{4 \pi\left(T_{2^{*}}-T_{1^{*}}\right) l_{*}}\left[\ln \left(\frac{t_{2 *}}{t_{1^{*}}}\right)\right]
$$

For $r_{*}=r_{w^{*}}$ the condition for the series truncation $r_{w^{*}}^{2} / 4 \alpha t_{*}<<1$ can be expressed in the following equivalent form that provides the validity condition of the approximation in the form

$t_{*}>t_{o^{*}}=\frac{r_{w^{*}}^{2}}{4 \alpha}$

The value of $t_{o^{*}}=r_{w^{*}}^{2} / 4 \alpha$ provides a validity condition of the experimental readings, i.e. $t_{*} \gg t_{o^{*}}$.

Equation (5) is a very accurate way of estimating the thermal conductivity as long as the validity conditions for appropriateness of the problem derivations used above are fulfilled. A finite length of the platinum (tantalum) wire, the finite size of the cylindrical container, the heat capacity of the platinum (tantalum) wire, and possibly natural convection effects are examples of possible deviations of any realistic system from the one used in deriving equation (5). De Groot et al. (1974), Healy et al. (1976), and Kestin \& Wakeham (1978) introduce an assessment of these deviations and possible corrections to the THW readings to improve the accuracy of the results. In general all the deviations indicated above could be eliminated via the proposed corrections provided the validity condition listed in equation (6) is enforced as well as an additional condition that ensures that natural convection is absent. The validity condition (6) implies the application of equation (5) for long times only. Nevertheless, when evaluating this condition (6) to data used in the nano-fluid suspensions experiments one obtains explicitly the following values. For a $76.2 \mu \mathrm{m}$ diameter of platinum wire used by Eastman et al. (2001), Lee et al. (1999), Choi et al. (2001) and an electrical insulation coating thickness of $10 \mu \mathrm{m}$, the wire radius is $r_{w^{*}}=4.81 \times 10^{-5} \mathrm{~m}$ leading to $t_{o^{*}}=r_{w^{*}}^{2} / 4 \alpha=13.7 \mathrm{~ms}$ for ethylene glycol and $t_{o^{*}}=r_{w^{*}}^{2} / 4 \alpha=7.2 \mathrm{~ms}$ for oil, leading to the validity condition $t_{*} \gg 13.7 \mathrm{~ms}$ for ethylene glycol and $t_{*}>7.2 \mathrm{~ms}$ for oil. The long times beyond which the solution (5) can be used reliably are therefore of the order of hundreds of milliseconds, not so long in the actual practical sense. These values also correspond to the ones needed for the assumption of a wire temperature that is uniform over the wire's cross section a condition that developed following eq.(1) above. On the other hand the experimental time range is limited from above as well in order to ensure the lack of natural convection that develops at longer time scales. Xuan \& Li (2000) estimate this upper limit for the time that an experiment may last before natural convection develops as about 5s. They indicate that "An experiment lasts about $5 \mathrm{~s}$. If the time is longer, the temperature difference between the hot-wire and the sample fluid increases and free convection takes place, which may result in errors" . Lee et al. (1999) while using the THW method and providing experimental data in the time range of $1 \mathrm{~s}$ to $10 \mathrm{~s}$, indicate in their figure 3 the "valid range of data reduction" to be between $3 \mathrm{~s}$ to $6 \mathrm{~s}$. Our estimations evaluated above confirm these lower limits as a very safe constraint and we assume that the upper limits listed by Xuan \& Li (2000) and Lee et al. (1999) are also good estimates. Within this time range the experimental results should produce a linear relationship, on a logarithmic time scale, between the temperature and time.

Fluid-Saturated Porous Media

The first major problem when attempting to apply the THW method to porous media is focused in the question "What temperature precisely does the wire is exposed to?". Obviously, the wire is exposed partly to the solid-phase and partly to the fluid phase that constitutes the porous medium. There is no justification to assume that local thermal equilibrium between the solid and fluid phases occurs generally (especially when a heat flux boundary condition is applied, as in the THW case). On the contrary, it is sensible to assume that the average temperature of the fluid differs from that of the solid. Then the wire being in contact with both phases will "feel" the fluid temperature on parts of its surface and the solid temperature on other parts of its surface. How to integrate these two effects in terms of its overall lumped effect on the total electrical resistance of the wire is not a simple averaging procedure. The following derivations demonstrate the direction we adopted in an attempt at resolving this problem. Obviously we need to separate between two extreme cases. One is related to the one extreme possibility when the pores near the wire form complete rings around the wire, i.e. at any point along the wire's length, the complete circumference of the wire is exposed either completely to the fluid or completely to the solid. In such a case one may look to this configuration as small electrical resistors connected in series along the wire's length. A macroscopic experimental testing of this concept is presented in Figure 3 where a two-phase system consisting of two different immiscible liquids is being used with the Transient Hot Wire system to check the following derivations.

Consider the two immiscible stationary liquids, a lighter one on top of a heavier one as presented in Figure 3. The total height of the container $H$ is occupied partly, $H_{d}$, by the heavy liquid on the down-section and partly, $H_{u}$, by the lighter liquid on the upper section. We define the relative height of the interface between the two fluids as $\phi=H_{d} / H$. Obviously, the following relationship holds $(1-\phi)=1-H_{d} / H=H_{u} / H$. Neglecting the tiny region in the neighborhood of the interface where sharp temperature gradients are being expected we can 
assume far away from the interface radial temperatures of the form that were presented for a single fluid in eq. (2)

$T_{d} \approx \frac{\dot{q}_{l^{*}}}{4 \pi k_{d}}\left[-\gamma_{E u}+\ln \left(\frac{4 \alpha_{d} t_{*}}{r_{w^{*}}^{2}}\right)\right]$
$T_{u} \approx \frac{\dot{q}_{l^{*}}}{4 \pi k_{u}}\left[-\gamma_{E u}+\ln \left(\frac{4 \alpha_{u} t_{*}}{r_{w^{*}}^{2}}\right)\right]$

leading to relationships of the form presented in eq.(5), i.e. the thermal conductivities of each liquid is approximately given by

$$
\begin{aligned}
& k_{d} \approx \frac{i V}{4 \pi\left(T_{d 2}-T_{d 1}\right) H}\left[\ln \left(\frac{t_{2^{*}}}{t_{1^{*}}}\right)\right] \\
& k_{u} \approx \frac{i V}{4 \pi\left(T_{u 2}-T_{u 1}\right) H}\left[\ln \left(\frac{t_{2^{*}}}{t_{1^{*}}}\right)\right]
\end{aligned}
$$

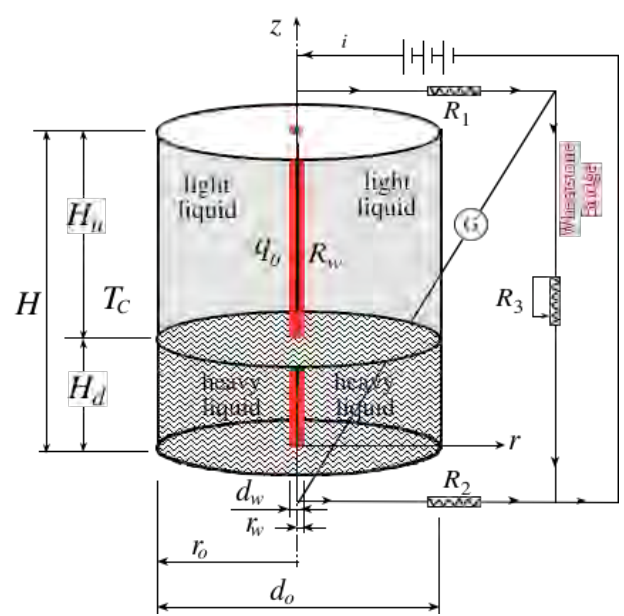

Figure 3 Schematic setup for a Transient Hot Wire experiment in two immiscible Fluids.

However, we do not measure separately the temperatures $T_{d}$ and $T_{u}$ but rather the wire's electrical resistance due to the lumped effect of both $T_{d}$ and $T_{u}$. The resistance of the wire is directly proportional to the wire's length and therefore the contribution of the lower and upper sections can be expressed in the form

$R_{w}=\phi R_{w d}+(1-\phi) R_{w u}$

where

$R_{w d}=R_{o}\left[1+\beta\left(T_{d}-T_{o}\right)\right]$ and $R_{w u}=R_{o}\left[1+\beta\left(T_{u}-T_{o}\right)\right]$

Combining (10) with (9) yields

$$
\begin{aligned}
R_{w}= & R_{o}\left\{1+\beta\left[\phi T_{d}+(1-\phi) T_{u}-T_{o}\right]\right\}= \\
R_{o} & {\left[1+\beta\left(T_{e f f}-T_{o}\right)\right] }
\end{aligned}
$$

where an effective temperature in the form

$T_{\text {eff }}=\phi T_{d}+(1-\phi) T_{u}$

appears and represents the average temperature "felt" by the wire in the sense of its impact on the wire's electrical resistance. Now, however we can express this effective temperature by using the individual temperatures (7) into (12) leading to

$T_{e f f} \approx \frac{\dot{q}_{l^{*}}}{4 \pi}\left[-\gamma_{E u}\left(\frac{\phi}{k_{d}}+\frac{(1-\phi)}{k_{u}}\right)+\frac{\phi}{k_{d}} \ln \left(\frac{4 \alpha_{d} t_{*}}{r_{w^{*}}^{2}}\right)+\frac{(1-\phi)}{k_{u}} \ln \left(\frac{4 \alpha_{u^{*}} t_{*}}{r_{w^{*}}^{2}}\right)\right]$

Evaluating eq.(13) at two instances of time $t_{1^{*}}$ and $t_{2^{*}}$ and evaluating the difference yields

$\left(T_{\text {eff }, 2}-T_{e f f, 1}\right) \approx \frac{\dot{q}_{l^{*}}}{4 \pi}\left[\frac{\phi}{k_{d}}+\frac{(1-\phi)}{k_{u}}\right] \ln \left(\frac{t_{2^{*}}}{t_{1^{*}}}\right)$

where an effective thermal conductivity emerged (not necessarily the typical "effective" value), in the form of thermal resistances $\left(1 / k_{i} \forall i=d, u\right)$ connected in series

$\frac{1}{k_{e f f}}=\frac{\phi}{k_{d}}+\frac{(1-\phi)}{k_{u}}$

This result is still not satisfactory because our aim is to measure $k_{u}$ and $k_{d}$ separately and not their lumped effect on the wire. To overcome this problem we need to run the experiment twice, with different values of the interface location, i.e. $\phi_{1}$ and $\phi_{2}$. Then, from the known effective values of $k_{\text {eff,1 }}$ and $k_{\text {eff }, 2}$ obtained by the method presented above one may solve the system of two equations

$\phi_{1} R_{d}+\left(1-\phi_{1}\right) R_{u}=R_{e f f, 1} ; \phi_{2} R_{d}+\left(1-\phi_{2}\right) R_{u}=R_{e f f, 2}$

where $R_{d}=1 / k_{d}, \quad R_{u}=1 / k_{u}$ and $R_{\text {eff }, i}=1 / k_{\text {eff }, j} \quad \forall j=1,2$

leading to

$k_{d}=\frac{\left(\phi_{1}-\phi_{2}\right)}{\left[\left(1-\phi_{1}\right) R_{e f f, 1}-\left(1-\phi_{2}\right) R_{e f f, 2}\right]} ; k_{u}=\frac{\left(\phi_{1}-\phi_{2}\right)}{\left[\phi_{1} R_{e f f, 2}-\phi_{2} R_{e f f, 1}\right]}$

Alternatively, one may use a second wire located far away (in the sense of the thermal impact of the heating from the wire, approximately $>>100 \mu m$ apart) from the first one and embedded only in the upper liquid, hence evaluating $k_{u}$ independently and then substituting it into (15) to obtain the value of $k_{d}$. More difficult is the other extreme when we assume that the wire is exposed partly to the fluid and partly to the solid but this separation is along the wire, i.e. part of circumference of any cross section, say $0<\theta_{*}<\theta_{1}$, is exposed to one fluid and the other part, $\theta_{1}<\theta_{*}<2 \pi$, is exposed to the other fluid where two partitions located at $\theta_{*}=0$ and $\theta_{*}=\theta_{1}$ separate the two fluids. In this case the cross section will not have a uniform temperature and while this configuration represents electrical resistances connected in parallel the very dependence of the temperature solution within the wire on $r_{*}$ and $\theta_{*}$ makes the application of the electrical resistance temperature relationship questionable. The realistic porous media outcome is expected to be in between these two limits and will depend on the areal-porosity of the porous medium and its distribution around and along the wire. More work will be needed to find precisely how to characterize a porous medium in a way that these parameters will be established 
independently and could then be used with confidence with the Transient Hot Wire method.

\section{Fluid-Suspensions}

The corresponding problem applied to fluid suspensions focusing primarily on nanofluids can be dealt with as a particluar case of the porous medium problem by removing the macroscopic level conduction mechanism representing the heat transfer within the solid phase because the solid particles represent the dispersed phase in the fluid suspension and therefore the solid particles can conduct heat between themselves only via the neighboring fluid. Substituting $k_{s}=0$ in the system of governing equations accomplishes this goal. The distinction between the porous medium and a fluid suspension is that in a porous medium both phases (solid and fluid) are interconnected, while in fluid suspensions only the fluid phase is interconnected while the solid suspended particles represent the dispersed phase. While the problems raised for the porous medium apply equally well for suspensions, the latter are expected to impact substantially less the measurement outcome because the typical solid fraction used in nanofluids is about a few percents, compared to over $70 \%-90 \%$ solid fraction (10\%-30\% porosity) in typical porous media.

\section{Interface heat transfer coefficient in porous media and fluid suspensions}

In addition to the important question raised above, and to compensate for the possibility that such an accurate representation of the "effective thermal conductivity" has its limitations, the averaging concept can be applied (in porous media the latter is common practice) by defining a Representative Elementary Volume (REV) and averaging the dependent variables over this REV. The resulting effect in addition to the heat transferred within each phase is the heat conduction over the interface separating the two phases (interphase heat transfer). Evaluating this heat transfer is possible for regular geometries, like the spherical one used by Maxwell (1891), however it becomes increasingly more difficult as the geometry becomes more complicated. Heat conduction in porous media subject to Lack of Local Thermal Equilibrium (LaLotheq) is governed at the macro-level by the following equations that represent averages over each phase within an REV (Representative Elementary Volume)

$\gamma_{s} \frac{\partial T_{s}}{\partial t_{*}}=k_{s} \nabla_{*}^{2} T_{s}-Q_{s f}$ (a) $\gamma_{f} \frac{\partial T_{f}}{\partial t_{*}}=k_{f} \nabla_{*}^{2} T_{f}+Q_{s f}$ (b)

where $Q_{s f}$ represents the rate of heat generation in the fluid phase within the REV due to the heat transferred over the fluidsolid interface, and where $\gamma_{s}=(1-\phi) \rho_{s} c_{s}$ and $\gamma_{f}=\phi \rho_{f} c_{p, f}$ are the solid phase and fluid phase effective heat capacities, respectively, $\phi$ is the porosity, $k_{s}=(1-\phi) \tilde{k}_{s}$ and $k_{f}=\phi \tilde{k}_{f}$ are the effective thermal conductivities of the solid and fluid phases, respectively. The traditional formulation of the rate of heat generation in the fluid phase within the REV due to the heat transferred over the fluid-solid interface uses a linear relationship between $Q_{s f}$ and the average temperature difference between the phases in the form $Q_{s f}=h\left(T_{s}-T_{f}\right)$. The coefficient $h>0$, carrying units of $\mathrm{W} \mathrm{m}-3 \mathrm{~K}-1$, is a macro-level integral heat transfer coefficient for the heat conduction at the fluid-solid interface (averaged over the REV) that is assumed independent of the phases' temperatures and independent of time. Note that this coefficient is conceptually distinct from the convection heat transfer coefficient and is anticipated to depend on the thermal conductivities of both phases as well as on the surface area to volume ratio (specific area) of the medium (Vadasz, 2006).

The lack of macroscopic level conduction mechanism in fluid suspensions (with $\varepsilon=(1-\phi)$ ) i.e. the heat transfer within the solid phase which is expressed by the fact that the solid particles represent the dispersed phase in the fluid suspension and therefore the solid particles can conduct heat between themselves only via the neighboring fluid, leads to setting $k_{s}=0$ in eq.(18a). The latter yields from $(18 \mathrm{a}, \mathrm{b})$ the averaged equations applicable to fluid suspensions. When steady state is accomplished in fluid suspensions $\partial T_{s} / \partial t_{*}=\partial T_{f} / \partial t_{*}=0$ leading to local thermal equilibrium between the solid and fluid phases, i.e. $T_{s}(\boldsymbol{r})=T_{f}(\boldsymbol{r})$, a condition that does not necessarily apply in porous media. Equations $(18 \mathrm{a}, \mathrm{b})$ are linearly coupled and represent the traditional form of expressing the process of heat conduction in porous media subject to LaLotheq (Nield and Bejan 2006, Nield 1998, 2002, Nield, Kuznetsov \& Xiong 2002, Lage 1999, Minkowycz, Haji-Shiekh \& Vafai 1999). By using (18a,b) for a line heat source according to the THW method and introducing the dimensionless variables $t=t_{*} h / \gamma_{f}, \quad r^{2}=r_{*}^{2} h / k_{f}$, $\theta_{s}=\left(T_{s}-T_{o}\right) /\left(\dot{q}_{l^{*}} / 2 \pi k_{f}\right), \theta_{f}=\left(T_{f}-T_{o}\right) /\left(\dot{q}_{l^{*}} / 2 \pi k_{f}\right)$ renders these equations into their corresponding dimensionless form

$$
\begin{aligned}
& \frac{1}{\chi} \frac{\partial \theta_{s}}{\partial t}=\frac{1}{\sigma r} \frac{\partial}{\partial r}\left(r \frac{\partial \theta_{s}}{\partial r}\right)-\left(\theta_{s}-\theta_{f}\right) ;(a) \\
& \frac{\partial \theta_{f}}{\partial t}=\frac{1}{r} \frac{\partial}{\partial r}\left(r \frac{\partial \theta_{f}}{\partial r}\right)+\left(\theta_{s}-\theta_{f}\right) \quad \text { (b) }
\end{aligned}
$$

where $\chi=\gamma_{f} / \gamma_{s}, \quad \sigma=k_{f} / k_{s}$, and $\kappa=\alpha_{f} / \alpha_{s}=\sigma / \chi$ represent the heat capacities, thermal conductivities and thermal diffusivities ratios, respectively. Introducing a Boltzmann transformation in the form

$\eta=\frac{r^{2}}{4 t}=\frac{r_{*}^{2}}{4 \alpha_{f} t_{*}}$

transforms eqs. (19 a,b) into the following equivalent, but not self-similar, form

$$
\begin{aligned}
t \frac{\partial \theta_{s}}{\partial t} & =\kappa^{-1} \frac{\partial}{\partial \eta}\left(\eta \frac{\partial \theta_{s}}{\partial \eta}\right)+\eta \frac{\partial \theta_{s}}{\partial \eta}-\chi t\left(\theta_{s}-\theta_{f}\right) ; \\
t \frac{\partial \theta_{f}}{\partial t} & =\frac{\partial}{\partial \eta}\left(\eta \frac{\partial \theta_{s}}{\partial \eta}\right)+\eta \frac{\partial \theta_{s}}{\partial \eta}+t\left(\theta_{s}-\theta_{f}\right)
\end{aligned}
$$


The corresponding single-phase equation is self-similar, a result of utmost importance because it is this self-similarity that produces the simple solution expressed by eq. (2) and makes the application of the THW method possible. Nevertheless, equations (21) produce interesting self-similar solutions for short times $t<<1$, (and we will see that these are precisely the times that the THW method is focused on), by using the following short times expansion $\theta_{i}=\theta_{i}^{(0)}+t \theta_{i}^{(1)}+t^{2} \theta_{i}^{(2)}$ $+O\left(t^{3}\right) \forall i=s, f$. Then the equations at leading order are $\kappa^{-1} \frac{\mathrm{d}}{\mathrm{d} \eta}\left(\eta \frac{\partial \theta_{s}^{(0)}}{\partial \eta}\right)+\eta \frac{\mathrm{d} \theta_{s}^{(0)}}{\mathrm{d} \eta}=0 ;(\mathrm{a})$

$\frac{\mathrm{d}}{\mathrm{d} \eta}\left(\eta \frac{\partial \theta_{f}^{(0)}}{\partial \eta}\right)+\eta \frac{\mathrm{d} \theta_{f}^{(0)}}{\mathrm{d} \eta}=0$

hence restoring at leading order the single-phase self-similarity at short times despite the dual-phase nature of the problem. The solution to $(22 \mathrm{a}, \mathrm{b})$ subject to the hot wire boundary conditions is presented below after converting it back to dimensional form

$$
\begin{aligned}
& \left(T_{s}^{(0)}-T_{o}\right)=\frac{\dot{q}_{l^{*}}}{4 \pi k_{s}}\left[-\gamma_{E u}+\ln \left(\frac{4 \alpha_{s} t_{*}}{r_{*}^{2}}\right)+O\left(\frac{r_{*}^{2}}{4 \alpha_{s} t_{*}}\right)\right] \\
& \left(T_{f}^{(0)}-T_{o}\right)=\frac{\dot{q}_{l^{*}}}{4 \pi k_{f}}\left[-\gamma_{E u}+\ln \left(\frac{4 \alpha_{f} t_{*}}{r_{*}^{2}}\right)+O\left(\frac{r_{*}^{2}}{4 \alpha_{f} t_{*}}\right)\right]
\end{aligned}
$$

These solutions can be used to evaluate the effective thermal conductivity of the solid and fluid phases respectively for any pairs of two temperature readings $\left(T_{f 1}^{(0)}, T_{s 1}^{(0)}\right)$ and $\left(T_{f 2}^{(0)}, T_{s 2}^{(0)}\right)$ taken at subsequent times $t_{*_{1}}$ and $t_{*_{2}}$, respectively, in the form

$$
\begin{aligned}
& k_{s} \approx \frac{\dot{q}_{l^{*}}}{4 \pi\left(T_{s 2}^{(0)}-T_{s 1}^{(0)}\right)}\left[\ln \left(\frac{t_{2^{*}}}{t_{1^{*}}}\right)\right] \\
& k_{f} \approx \frac{\dot{q}_{l^{*}}}{4 \pi\left(T_{f 2}^{(0)}-T_{f 1}^{(0)}\right)}\left[\ln \left(\frac{t_{2^{*}}}{t_{1^{*}}}\right)\right]
\end{aligned}
$$

These results are quite promising because they indicate that the THW method may be applied with some higher order corrections in porous media too, however we need to check the conditions under which the latter applies. Clearly a condition similar to eq.(6) which is applicable to single-phase applies here too as the truncation of (23) and (24) requires $t_{*}>>t_{o}$, where $t_{o}=\max \left[\left(r_{w^{*}}^{2} / 4 \alpha_{f}\right),\left(r_{w^{*}}^{2} / 4 \alpha_{s}\right)\right]$. In addition, the leading order solutions (23) and (24) apply for dimensionless short times only, i.e. for $t=t_{*} h / \gamma_{f}<<1$. Note also that an inherent, but reasonable, assumption was included in the derivation of the leading order equations (23) and (24), implying that $\chi=\gamma_{f} / \gamma_{s}=O(1)$, i.e. $\gamma_{f} \sim \gamma_{s}$ at least in their order of magnitude. Therefore the condition $t=t_{*} h / \gamma_{f}<<1$ implies $t_{*}<<\gamma_{f} / h$ or similarly $t_{*}<<\gamma_{s} / h$. These conditions specify the range of short times that are needed for the approximated solutions (23) and (24) leading to (25) to be valid. Combining the two conditions above produces

$t_{o}<<t_{*}<<t_{m}$

where

$t_{o}=\max \left[\left(r_{w^{*}}^{2} / 4 \alpha_{f}\right),\left(r_{w^{*}}^{2} / 4 \alpha_{s}\right)\right] ; t_{m}=\min \left[\left(\gamma_{f} / h\right),\left(\gamma_{s} / h\right)\right]$.

The following requirement is necessary and sufficient for such a time interval to exist and makes the THW experimental method applicable to dual-phase systems, in the form: $t_{m}>>t_{o}$. This requirement implies $\gamma_{f} / h>>r_{w^{*}}^{2} / 4 \alpha_{f}$ (assuming $\chi=\gamma_{f} / \gamma_{s}=O(1)$ ), leading to

$h<<\frac{4 k_{f}}{r_{w^{*}}^{2}}$

This condition is the requirement for the existence of a time interval over which the THW method may produce reliable results in Dual-Phase applications. It reveals the significant impact that the interface heat transfer coefficient $h$ has on the applicability of the THW method to dual-phase systems.

In fluid suspensions like in porous media, the parameter $h$, carrying units of $\mathrm{W} \cdot \mathrm{m}^{-3} \cdot \mathrm{K}^{-1}$, represents an integral heat transfer coefficient for the heat conduction at the solid-fluid interface within an REV. Its general relationship to the surfacearea-to-volume ratio (specific area) was derived by Vadasz (2006) by using relationships that are available for the respective coefficient in fluid saturated porous media (Quintard \& Whitaker 1995, Alazmi \& Vafai 2002, Amiri \& Vafai 1994, Wakao, Kaguei \& Funazkri 1979, Wakao \& Kaguei 1982, Kuwahara, Shirota \& Nakayama 2001). Most of the reported evaluations of $h$ in porous media listed above were derived with a particular focus on convection rather than conduction heat transfer. Their applicability and accuracy for conduction are therefore questionable. The implication of the derived relationship presented by Vadasz (2006) is that the heat transfer coefficient is related to the particle size by the inversely quadratic relationship $h=\left[k_{f} / d_{p}^{2}\right] s\left(\varepsilon, k_{f} / k_{s}\right)$. While the particular form of the function $s\left(\varepsilon, k_{f} / k_{s}\right)$ and its possible further dependence on the particle size as well, especially as the particle size is reduced to nano-scale levels, is not established for the case of suspensions, the general dependence of the heat transfer coefficient on the particle size is evident. This dependence of the heat transfer coefficient on the particle size introduces the effect of the surface-area-to-volume ratio (specific area) that was claimed by Eastman et al. (2001) to be missing in the classical models of evaluating the effective thermal conductivity of the suspension. One should however bear in mind that further dependence on particle size is anticipated as the particle size is reduced to the nano-scale level predominantly when the ballistic rather than diffusive nature of heat transfer becomes dominant (Chen 1996, 2000, 2001) and hence reducing the rate of heat transfer, implying a consequential reduction of the value of $h$ to somewhat compensate for the otherwise substantial increase of $h$ as the particle size is reduced. In addition one may anticipate an increase of $h$ due to Brownian motion induced nanoconvection 
Jang and Choi (2004), Prasher, Bhattacharya and Phelan, (2005) or a decrease due to the exceptionally small interface thermal conductance Huxtable et al. (2003).

\section{CONCLUSIONS}

An attempt to render the Transient Hot Wire Experimental method to dual-phase applications has been presented. Substantial more work is however necessary to complete this process and produce clear validity criteria for such applications. The latter criteria will then be used to develop reliable experimental procedures within these validity limits.

\section{REFERENCES}

[1] Alazmi, B. and Vafai, K., 2002, Constant Wall Heat Flux Boundary Conditions in Porous Media under Local Thermal NonEquilibrium Conditions, International Journal of Heat and Mass Transfer, 45, 3071-3087.

[2] Amiri, A. \& Vafai, K., 1994, Analysis of dispersion effects and non-thermal equilibrium, non-Darcian, variable porosity incompressible flow through porous media, Int. J. Heat Mass Transfer, 37, 934-954.

[3] Assael, M.J., Dix, M., Gialou, K., Vozar, L. \& Wakeham, W.A., 2002, Application of the Transient Hot-Wire technique to the measurement of the thermal conductivity of solids, Int. J. Thermophysics, 23, 615-633.

[4] Assael, M.J., Chen, C.-F., Metaxa, I., \& Wakeham, W.A., 2004, Thermal conductivity of suspensions of carbon nanotubes in water, Int. J. Thermophysics, 25, 971-985.

[5] Bentley, J.P., 1984, Temperature sensor characteristics and measurement system design, Journal of Physics E: Scientific Instruments, 17, 430-439.

[6] Chen, G., 1996, Nonlocal and nonequilibrium heat conduction in the vicinity of nanoparticles, ASME J. Heat Transfer, 118, 539545.

[7] Chen, G., 2000, Particularities of heat conduction in nanostructures, Journal of Nanoparticle Research, 2, 199-204.

[8] Chen, G., 2001, Balistic-diffusive heat-conduction equations, Physical Review Letters, 86 (11), 2297-2300.

[9] Choi, S.U.S., Zhang, Z.G., Yu, W., Lockwood, F.E., \& Grulke, E.A., 2001, Anomalous thermal conductivity enhancement in nanotube suspensions, Appl. Physics Letters, 79, 2252-2254.

[10] Coquard, R., Bailis, D., 2006, Modeling of heat transfer in lowdenisty EPS foams, ASME J. Heat Transfer, 128, 538-549.

[11] Coquard, R., Bailis, D., Quenard, D., 2006, Experimental and theoretical study of the hot-wire method applied to low-density thermal insulators, Int. J. Heat Mass Trasnfer, 49, 4511-4524.

[12] De Groot, J.J., Kestin, J. \& Sookiazian, H., 1974, Instrument to measure the thermal conductivity of gases, Physica, 75, 454-482.

[13] Eastman, J.A., Choi, S.U.S., Li, S., Yu, W., \& Thompson, L.J., 2001, Anomalously increased effective thermal conductivities of ethylene glycol-based nanofluids containing copper nanoparticles, Appl. Physics Letters, 78, 718-720.

[14] Hammerschmidt, U. \& Sabuga, W., 2000, Transient Hot Wire (THW) method: Uncertainty assessment, Int. J. Thermophysics, 21, 1255-1278.

[15] Healy, J.J., de Groot, J.J. \& Kestin, J., 1976, The theory of the Transient Hot-Wire method for measuring thermal conductivity, Physica, 82C, 392-408.

[16] Huxtable, S.T., Cahill, D.G., Shenogin, S., Xue, L., Ozisik, R., Barone, P., Usrey, M., Strano, M.S., Siddons, G., Shim, M., and Keblinski, P., 2003, Interfacial heat flow in carbon nanotube suspensions, Nature Materials, 2, 731-734.
[17] Jang, S.P., \& Choi, S.U.-S., 2004, Role of Brownian motion in the enhanced thermal conductivity of nanofluids, Applied Physcis Letters, 84 (21), 4316-4318.

[18] Kestin, J., \& Wakeham, W.A., 1978, A contribution to the theory of the transient hot-wire technique for thermal conductivity measurements, Physica, 92A, 102-116.

[19] Kuwahara, F., Shirota, M. \& Nakayama, A., 2001, A numerical study of interfacial convective heat transfer coefficient in twoenergy equation model for convection in porous media, Int. J. Heat Mass Transfer, 44, 1153-1159.

[20] Lage, J.L., 1999, The Implications of the Thermal Equilibrium Assumption for Surrounding-driven Steady Conduction within a Saturated Porous Medium Layer, International Journal of Heat and Mass Transfer 42, 477-485.

[21] Lee, S., Choi, S.U.-S., Li, S., \& Eastman, J.A., 1999, Measuring thermal conductivity of fluids containing oxide nanoparticles, ASME J. Heat Transfer, 121, 280-289.

[22] Martinsons, C., Levick, A., Edwards, G., 2001, Precise measurements of thermal diffusivity by photothermal radiometry for semi-infinite targets using accurately determined boundary conditions, Analytical Sciences, 17, 114-117.

[23] Maxwell, J.C., 1891, A Treatise on Electricity and Magnetism, 3rd edition, Clarendon Press, 1954 reprint, Dover, New York, pp. 435-441.

[24] Minkowycz, W.J., Haji-Shiekh, A., and Vafai, K., 1999, On Departure from Local Thermal Equilibrium in Porous Media Due to a Rapidly Changing Heat Source: the Sparrow Number, International Journal of Heat and Mass Transfer 42, 3373-3385.

[25] Nagasaka, Y. \& Nagashima, A., 1981, Absolute measurement of the thermal conductivity of electrically conducting liquids by the transient hot-wire method, J. Phys. E: Sci. Instrum., 14, 14351440 .

[26] Nield, D. \& Bejan, A., 2006, Convection in Porous Media, 3rd edition, Springer, New York.

[27] Nield, D.A., 2002, A Note on the Modeling of local Thermal Non-Equilibrium in a Structured Porous Medium International Journal of Heat and Mass Transfer 45, 4367-4368.

[28] Nield, D.A., Kuznetsov, A.V. and Xiong, M., 2002, Effect of Local Thermal Non-Equilibrium on Thermally Developing Forced Convection in a Porous Medium, International Journal of Heat and Mass Transfer 45, 4949-4955.

[29] Nield, D.A., 1998, Effects of local thermal nonequilibrium in steady convective processes in a saturated porous medium: forced convection in a channel, J. Porous Media, 1, 181-186.

[30] Prasher, R., Bhattacharya, P. \& Phelan, P. E., 2005, Thermal conductivity of nanoscale colloidal solutions (Nanofluids), Physical Review Letters, 94, 025901(1)-025901(4).

[31] Quintard, M., and Whitaker, S., 1995, Local Thermal Equilibrium for Transient Heat Conduction: Theory and Comparison with Numerical Experiments, International Journal of Heat and Mass Transfer 38, 2779-2796.

[32] Vadasz, P., 2006, Heat Conduction in Nanofluid Suspensions, Journal of Heat Transfer 128, 465-477.

[33] Wakao, N., Kaguei, S. \& Funazkri, T., 1979, Effect of fluid dispersion coefficients on particle-to-fluid heat transfer coefficients in packed beds, Chem. Engng. Sci., 34, 325-336.

[34] Wakao, N. \& Kaguei, S., 1982, Effect of fluid dispersion coefficients on particle-to-fluid heat transfer coefficients in packed beds, Heat and Mass Transfer in Packed Beds, Gordon and Breach, New York.

[35] Xuan, Y. \& Li, Q., 2000, Heat transfer enhancement of nanofluids, Int. J. Heat Mass Transfer, 21, 58-64. 